\title{
COMMUNICATIONS
}

\section{EXPERIMENTAL TRACHOMA PRODUCED BY A WEST AUSTRALIAN VIRUS*}

\author{
BY
}

IDA MANN, $\ddagger$ C. H. GREER, $\uparrow$ DOROTHY PERRET, $\ddagger$ AND C. MCLEAN

From the $\ddagger$ Department of Public Health, Perth, Western Australia, and the $\dagger$ Lions National Research Unit of the Victorian Eye and Ear Hospital, Melbourne, Victoria

RECENT ophthalmic surveys in Western Australia by Mann (1954a, 1954b, 1956), with the support of the Public Health Department and the Royal Flying Doctor Service, have shown that trachoma is the greatest single cause of ocular morbidity and blindness over large areas of rural Australia. The first successful culture in Australia of the causative agent was accomplished in Perth by Perret and Mann (1960). The virus was obtained by scraping the conjunctiva of a 3-year-old half-caste boy in Moora, a small town about 100 miles north of Perth. This child showed typical necrotic follicles in the upper tarsal conjunctiva but had no discharge and was quite asymptomatic. The virus was passaged through eggs according to the method of T'ang, Chang, Huang, and Wang (1957). This Australian virus behaved culturally in a similar way to the virus isolated from Gambian trachoma by Collier and Sowa (1958). The present communication deals with the further investigation of this same strain of Australian trachoma virus and the production by it of clinical trachoma in a human volunteer.

\section{Preparation and Testing of the Moora Virus}

All chick-embryo inoculations were made by the yolk-sac route using an inoculum of $0.25 \mathrm{ml}$. into 7- or 8-day embryos. Yolk-sac smears were stained by Macchiavello's method. Yolk-sac suspensions were prepared by grinding the yolk sacs in a mortar cooled to $-15^{\circ} \mathrm{C}$. and making up into 20 per cent. (w/v) suspension with saline containing 10 per cent. broth and 20,000 U/ml. streptomycin. Yolk sacs or yolk-sac suspensions were tested routinely for bacteriological sterility by inoculating serum broth and cooked-meat medium.

Inoculation of Rhesus Monkey.-A suspension made from the yolk sacs of two embryos dying on the 8th day in the fifth passage of the virus was stored at $4^{\circ} \mathrm{C}$. for $1 \mathrm{hr}$. and then used to inoculate a Rhesus monkey. A cotton wool swab dipped in the suspension was rubbed firmly on the upper tarsal conjunctiva of both eyes. 
Examinations of the everted eyelids were made at intervals of 2-6 days for 6 weeks and then at 8 and 12 weeks. Hyperaemia was first detected on the 12th day and was also observed at each subsequent examination up to the eighth week. On the 18th day, four small pale follicles were seen at the edges of the tarsal plates, three on the left, and one on the right. These follicles appeared to be smaller on the 25 th day and had disappeared by the 31 st day. Follicles were again observed on the 37 th day (three on the margin of the right tarsal plate, and one, or possibly two, on the left) but had disappeared by the 42 nd day. At the final examination, made 12 weeks after the inoculation, both tarsal plates looked quite normal. Thus, as is usually the case in monkeys, only mild follicular conjunctivitis was produced without either corneal change or pannus and without post-follicular scarring.

Immediately before inoculating the monkey, the same suspension was inoculated into four 7-day embryos. One embryo died after 3 days and the others after 7 days. Yolk-sac smears from the embryos dying after 7 days showed bodies with the typical appearance of elementary bodies of the psittacosis-lymphogranuloma venereum (P-LGV) group of viruses.

Suspension for Use in the Human Volunteer.-The yolk sac of an embryo dying on the 8th day in the eleventh passage of the virus, and showing intracellular elementary bodies typical of the P-LGV group of viruses, was stored overnight at $4^{\circ} \mathrm{C}$. while preliminary tests of its bacteriological sterility were carried out. On the following day the suspension was prepared and divided into two portions which were both stored at $0^{\circ} \mathrm{C}$.; one portion was flown to Melbourne and the other retained in Perth. 18 hours later, at about the time of the human inoculation, the retained portion of the suspension was inoculated undiluted and at dilutions of $10^{-1}, 10^{-2}$, and $10^{-3}$, into 8-day chick embryos, using four embryos per dilution. Three embryos inoculated with the undiluted suspension died after 8,11 , and 12 days respectively, and elementary bodies were seen in smears of their yolk sacs. All other embryos survived for 12 days and were not examined further. Both the yolk sac and the yolk-sac suspension were tested for the presence of bacteria by examination of Gram-stained and Giemsa-stained smears and by the inoculation of horse-blood agar plates (incubated aerobically and anaerobically), serum broth, cooked-meat medium, and sodium thioglycolate liquid medium. Since growth in the liquid media could not be detected by inspection because of the turbidity of the inoculum, Gram-stained smears were examined after these media had been incubated at $37^{\circ} \mathrm{C}$. for 1,2 , and 5 days. Sub-cultures to fresh media of the same type and to blood-agar were made at 2 and 5 days. No bacteria were detected by these tests.

The yolk-sac-streptomycin suspension for the control volunteer was made from the yolk sac of a live 15-day chick embryo and prepared, stored, and tested for bacteriological sterility in exactly the same way as the virus suspension for the volunteer.

\section{Clinical Trial}

The live virus suspension and the control suspension were flown at $0^{\circ} \mathrm{C}$. from Perth to Melbourne for the next part of the experiment, which was carried out at the Victorian Eye and Ear Hospital and the Lions National 
Research Unit. Two volunteers were obtained-one to receive live virus suspension, the other to receive the control yolk-sac-streptomycin suspension.

(1) Control Volunteer.-The patient was a healthy woman aged 76 years who had been blind in both eyes from the age of 14 . Blindness was said to have followed a chronic septic infection, but no detailed history was available. The right eye was staphylomatous with an opaque scarred cornea. A few vessels extended into the scar but there was no true pannus. The patient had at one stage been treated with copper sulphate and the upper tarsal conjunctiva of the right eye showed a typical scarring almost certainly due to treatment and not to trachoma. The left eye was represented by a fibrous nodule in the depth of the orbit. The socket was contracted and the lids could not be everted.

After bacterial cultures had been made from both sides, the control inoculum was rubbed on to the conjunctiva of the everted lids of the right eye after instillation of 5 per cent. cocaine. The patient noticed nothing except a slight sensation of grittiness which persisted till the next day. A slight sticky discharge was present on the 2nd day, and on the 3rd day the lid margins were slightly reddened. By the 4th day nothing unusual was detectable. Cultures and scrapings were taken on the 6th day.

(2) Test Volunteer.-This was a very fit and mentally alert old lady aged 87 years. Her history was one of chronic glaucoma simplex which had begun about 25 years ago. Both eyes had been trephined successfully, but 5 years ago cataract developed. Extraction of the right lens was followed by massive intra-ocular haemorrhage, and although the eye was not lost it did not recover perception of light. The left eye had an immature cataract, loss of the visual field on the nasal side, and a visual acuity equal to hand movements at $2 \mathrm{ft}$.

It was decided to use the right eye for the experiment, as its tension was not raised and, except for the operation scars, the cornea and limbus were normal. Before inoculation, bacterial cultures were made and serum taken for antibody estimation. The left eye was protected by a Buller's shield.

On the day of the experiment the right eye was cocainized ( 5 per cent.) and a cotton wool swab dipped in the virus suspension was rubbed firmly over the everted upper lid and along the lower fornix. The patient complained of intense itching lasting for 5-10 minutes.

Nothing was noticed till the early morning of the 6th day, when the patient said the eye was irritating and felt as if "something was running round in it". Very little was noted other than slight discharge and slight swelling of the lower lid. The upper tarsal conjunctiva was possibly very slightly hyperaemic. Later the same day the right pre-auricular gland was slightly tender on pressure.

On the 7th day it was obvious that an acute condition had developed (Fig. 1, overleaf). The lids were stuck together with muco-pus, the upper tarsal conjunctiva was hyperaemic with a few petechiae and the pre-auricular gland was palpable. A conjunctival scraping showed very numerous cytoplasmic inclusions in the epithelial cells (vide infra). 
By the 8th day, a minute follicle was visible on the edge of the tarsal plate and the lids were oedematous and hyperaemic (Fig. 2, overleaf). This state of acute inflammation persisted and the pre-auricular gland became enlarged and tender. Epithelial keratitis and epithelial bedewing developed in the upper part of the cornea on the 10th day, and small follicles appeared at the outer end of the tarsal plate. At first, the corneal epithelium did not stain with fluorescein but presented only a faintly greyish spotting. By the 13th day, however, punctate staining was evident all over the cornea and the upper and lower palpebral conjunctiva was finely granular all over. A further scraping, taken on the 12th day, again showed cytoplasmic inclusions. Chemosis, especially of the lower part of the bulbar conjunctiva, became marked and there was great engorgement of all the limbal loops, especially above.

By the 15th day, the muco-purulent discharge had lessened to a slight watery exudate while the chemosis and pre-auricular adenopathy persisted. By the 21st day there were visible, with the slit lamp, nine minute new vascular loops arising from limbal vessels above and running downwards in the oedematous cornea. By the 24th day, eleven new vessels had appeared and pannus could be diagnosed with certainty. These vessels continued to increase in number and in length until the 35th day. They were exceedingly narrow loops, the entering and returning channels being so close together that, with a loupe alone, they could easily be mistaken for single downsprouts. It appears certain, however, that they were narrow loops from the beginning.

The acute phase of the disease began to subside before the appearance of the pannus so that by the 25 th day there was very little discomfort or discharge and the lids were no longer glued together in the morning.

On the 30th day it was decided to treat the patient, since the classical signs of trachoma had appeared after the expected incubation period of 6 to 7 days. Thus, hyperaemia, discharge, papillary hypertrophy, follicles, epithelial keratitis, pannus, pre-auricular adenopathy, and inclusion bodies of typical form had all been observed. In view of the patient's age and her wonderful co-operation, and of the fact that virus had been recovered in eggs on three occasions and passaged twice, it was thought wise to terminate the experiment. On the 31 st day, therefore, after serum, material for viral culture, a conjunctival scraping for microscopy, and a biopsy from the lower fornix had been taken, treatment was commenced. The regime was one tablet a day of Lederkyn by mouth and aureomycin ointment locally every 2 hours during the day.

Within 24 hours of starting treatment all discharge disappeared, the preauricular gland subsided, and the limbal vessels became much less engorged. The pannus vessels, however, continued to increase in length and punctate staining of the cornea persisted. The eye continued to improve, and, with the subsidence of the swelling, it became possible to see (with the slit lamp) innumerable tiny opaque spots just under the epithelium of the upper tarsal plate and of the lower fornix. These were possibly aggregates of lymphocytes and could be precursors of future follicles. Viral and bacterial cultures, a conjunctival scraping, and a blood sample were taken on the 38 th day. 
The clinical course of the first 38 days of the experiment is summarized in Table I.

TABLE I

SUMMARY OF CLINICAL COURSE

\begin{tabular}{|c|c|c|c|c|c|c|c|c|}
\hline $\begin{array}{l}\text { Day after } \\
\text { Inocula- } \\
\text { tion }\end{array}$ & $\begin{array}{l}\text { Discom- } \\
\text { fort }\end{array}$ & Discharge & $\begin{array}{l}\text { Conjunc- } \\
\text { tival } \\
\text { Injection }\end{array}$ & $\begin{array}{l}\text { Epithelial } \\
\text { Keratitis }\end{array}$ & $\begin{array}{l}\text { Papillary } \\
\text { Hyper- } \\
\text { trophy }\end{array}$ & Follicles & $\begin{array}{l}\text { Adeno- } \\
\text { pathy }\end{array}$ & Pannus \\
\hline D.6 & ++ & ++ & + & & & & + & \\
\hline D.7 & ++ & +++ & ++ & & & & ++ & \\
\hline D.8 & ++ & +++ & +++ & & & + & ++ & \\
\hline D.11 & ++ & ++ & ++++ & +++ & & ++ & +++ & Open limbal vessels \\
\hline D.15 & ++ & ++ & ++++ & +++ & +++ & ++ & +++ & Limbus engorged \\
\hline D. 17 & ++ & ++ & ++++ & ++++ & +++ & ++ & +++ & A few vessels? \\
\hline D. 21 & ++ & ++ & +++ & ++++ & +++ & ++ & + & Nine small vessels \\
\hline D. 24 & ++ & ++ & $+t+$ & ++++ & +++ & ++ & + & Eleven small vessels \\
\hline D. 27 & + & + & +++ & +++ & +++ & ++ & + & Fifteen vessels \\
\hline D.31 & $\begin{array}{c}\text { Treatment } \\
\text { started }\end{array}$ & + & +++ & ++++ & +++ & +++ & & Fifteen + vessels \\
\hline D. 33 & & & +++ & +++ & ++ & +++ & & $\begin{array}{l}\text { Intermittent flow in } \\
\text { vessels }\end{array}$ \\
\hline D. 38 & & & + & +++ & +++ & +++ & & $\begin{array}{l}\text { Still some circulating } \\
\text { blood }\end{array}$ \\
\hline
\end{tabular}

\section{Laboratory Investigations}

(1) Conjunctival Scrapings.-These were stained with iodine by the method of Gilkes, Smith, and Sowa (1958), and the presence of a carbohydrate matrix in the virus aggregations was confirmed. After decolorization with absolute methyl alcohol, the smears were restained for $1 \frac{1}{2}-2 \mathrm{hrs}$ with dilute Giemsa-stain (Gurr's R.66) - 1 drop (drop-bottle) to each $1 \mathrm{ml}$. of buffered water ( $\mathrm{pH}$ 6.8). Differentiation was done rapidly by immersion in absolute methyl alcohol for 2 sec., repeated, if necessary, to achieve the required tint.

On D.7 scrapings from the upper tarsal conjunctiva of the infected eye showed very numerous neutrophils and lymphocytes and abundant cellular debris. Many macrophages were also present, some showing cytoplasmic vacuoles and angular ingested fragments. Only an occasional eosinophil was seen. The epithelial cells contained very numerous inclusions in every way characteristic of trachoma. As many as eight large inclusions were seen in a single oil-immersion field (magnification $\times 950$ ). Particles of elementary body size, staining purple to red, formed helmet-shaped aggregations capping the nucleus, or large spherical aggregations in the cytoplasm. In addition, elementary bodies were seen to fill some cells and to be broadcast from ruptured cells. Spherical and oval, basophilic, initial bodies in one or more 
tight clusters were seen in many cells, while in others there were crescentic and spherical aggregates of mixed initial and elementary bodies. In the cytoplasm of a few epithelial cells, red to purple perinuclear networks were seen which were finer in texture than nuclear extrusions and appeared to resemble the reticulum described by Mori (1958) and regarded by him as provirus (Figs 3 to 6, opposite).

On D.12 the scrapings contained fewer inflammatory cells and noticeably fewer inclusions. On D.31, the day treatment was started, inclusions were still present in moderate numbers, but by D.36 only one recognizable aggregation of initial bodies was found. Many degenerate inflammatory cells were present together with moderate numbers of very large epithelial cells, possible evidence of epithelial regeneration. No virus inclusions were seen in scrapings from the control patient at any time.

(2) Conjunctival Biopsy.-On D.31 a small biopsy from the lower fornix showed that the conjunctival epithelium was thin, partly necrotic and infiltrated with neutrophils. No virus inclusions could be identified. The subepithelial stroma was much thickened by a dense inflammatory infiltration of plasmocytes, lymphocytes, and macrophages. Within this infiltration there were scattered, poorly demarcated foci of larger mononuclear cells in mitosis and macrophages with ingested basophilic particles (Leber cells). There was, however, no clearly defined follicular pattern.

(3) BACTERIAL Cultures.-These were made at intervals on aerobic blood agar plates and in infusion broth.

(a). Control.-Before inoculation of the control yolk-sac-streptomycin suspension, cultures from the right eye yielded $\alpha$-haemolytic streptococcus, Corynebacterium xerosis, and Staphylococcus albus, while the left socket grew, in addition, Haemophilus influenzae (Koch-Weeks bacillus). On the 6th day after inoculation only Staph. albus survived in the right eye (probably due to streptomycin in the inoculum) while the flora of the right socket remained unchanged.

(b) Test Volunteer.-Both before the inoculation of virus and on several occasions afterwards, cultures from the infected eye yielded only $C$. xerosis and Staph. albus; even at the height of the muco-purulent discharge, these were the only organisms isolated.

(4) RE-ISOLATION OF THE VIRUS.-Conjunctival scrapings from the test volunteer on D.11, D.17, and D.31 were suspended in $1 \mathrm{ml}$. saline containing 10 per cent. broth and 20,000 units streptomycin, and immediately flown back to Perth at $0^{\circ} \mathrm{C}$. About $12 \mathrm{hrs}$ after each scraping had been taken, the suspension was inoculated by the yolk-sac route into three 7-day embryos. From each of the scrapings an agent with the characteristics of the P-LGV 


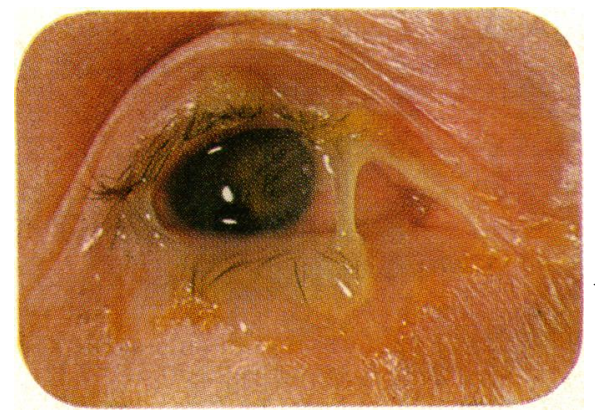

Fig. 1.-D.7: Acute muco-purulent conjunctivitis.

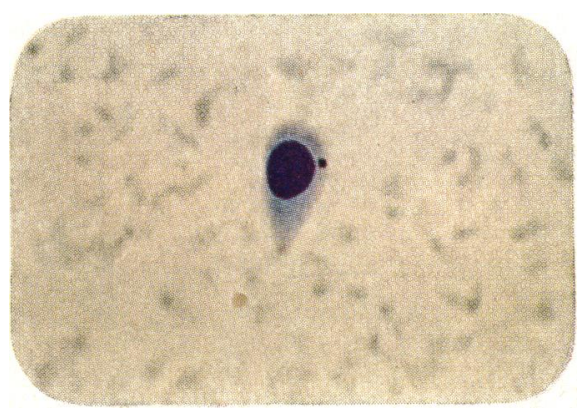

FIG. 3

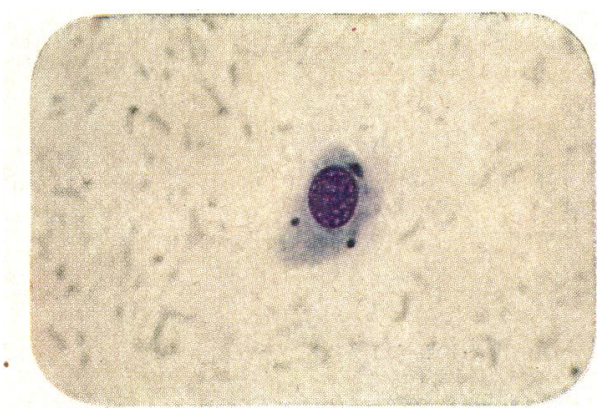

FIGs 3-5.-D.11 : Conjunctival scraping showing initial bodies in the cytoplasm of epithelial cells. Stained Giemsa. $\times 900$.

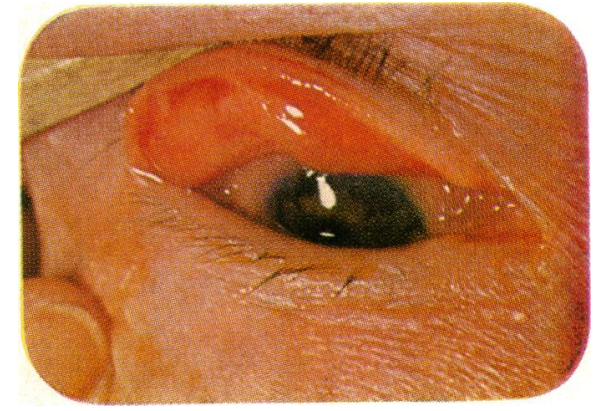

FIG. 2.-D.8: Conjunctival injection and oedema of lids. A minute follicle was present.

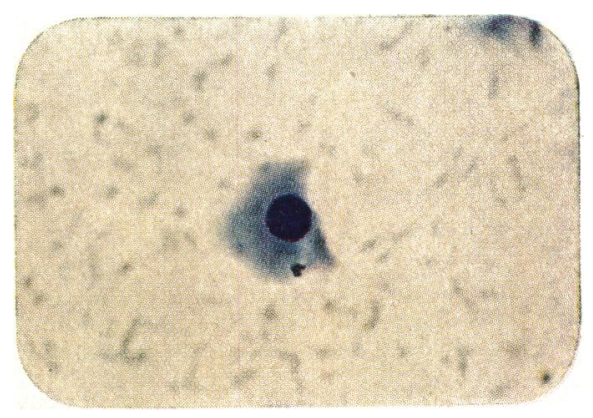

FIG. 4

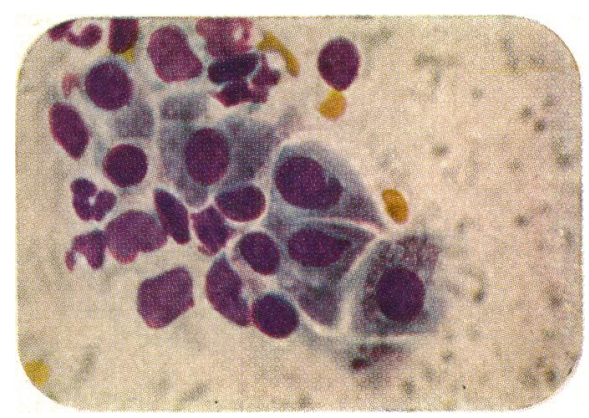

FIG. 6.-D.7: Conjunctival scraping showing a perinuclear network (bottom right) in an epithelial cell. At 11 o'clock from this cell, another epithelial cell contains a crescentic perinuclear aggregate of elementary bodies. Stained Giemsa. $\times 900$.

Facing page 646. 
group of viruses was isolated and passaged in the chick embryo. The timesto-death of the embryos of the early passages are shown in Table II. Yolksac smears from embryos dying later than the 4th day were stained by Machiavello's method and examined for elementary bodies of the P-LGV group of viruses. Typical intracellular and extracellular elementary bodies were seen in most of the smears from the primary passage, and were generally more numerous in smears from subsequent passages.

TABLE II

RE-ISOLATION OF THE VIRUS IN THE YOLK SAC OF THE CHICK EMBRYO

\begin{tabular}{c|c|c|c|c}
\hline \multirow{2}{*}{$\begin{array}{c}\text { Day } \\
\text { Scraping } \\
\text { Taken }\end{array}$} & Source & \multicolumn{3}{|c}{ Time (days) between Inoculation and Death of Embryo } \\
\cline { 3 - 5 } & & Passage 1 & Passage 2 & Passage 3 \\
\hline D.11 & (Upper Lid) & $6,7,12$ & $6,7,7,8,8,9$ & $4,9,9,9$ \\
D.17 & (Lower Lid) & $2,7,8$ & $2,6,8,9$ & $4,10,10,11,12,12$ \\
D.17 & (Upper Lid) & $2,8,9$ & $2,6,9,11$ & $7,8,9,9$ \\
D.31 & (Lower Lid) & $7,11,11$ & $7,9,10,12$ & \\
\hline
\end{tabular}

At the time of writing, the virus isolated from the scraping taken from the lower lid of the test volunteer on the 17th day has been through eleven passages; it has usually caused death in 6 to 10 days and typical elementary bodies have been seen at each passage.

No bacteria were detected in any smear, or in serum broth or cookedmeat medium inoculated with portions of yolk sac or yolk-sac suspensions at the end of each passage.

(5) Serological Investigations. - These were carried out in the Serology Section of the Public Health Laboratory Service of Western Australia.

One serum sample was taken from the test volunteer before inoculation and six samples at approximately weekly intervals thereafter. Serum was taken from the control volunteer before inoculation and at approximately 1 and 3 weeks after inoculation. These sera were tested for complementfixing antibodies using an ornithosis-lymphogranuloma venereum complement-fixing antigen. This was prepared by Commonwealth Serum Laboratories from a virus shown to be serologically related to the Moora virus (Perret and Mann, 1960). The Moora virus itself and the virus reisolated from the test volunteer were not available for use as antigens.

Tests were incubated at $4^{\circ} \mathrm{C}$. overnight to allow complement fixation to occur; otherwise the technique used was that described by Meyer and Eddie (1956). A positive human serum from a known case of psittacosis was included in the test. Altogether seven serum samples from this patient and three from the control were tested. Complement-fixing antibodies were not detected in any of the serum samples. 


\section{Summary}

The clinical and laboratory features of experimental trachoma in a human volunteer are described. The virus was obtained in Western Australia from an asymptomatic half-caste boy with necrotic follicles in his upper tarsal conjunctiva, and was passaged in embryonate eggs. An infected volunteer developed muco-purulent conjunctivitis, epithelial keratitis, pre-auricular adenitis, small granular follicles, pannus, and typical cytoplasmic inclusions. The virus was recovered from conjunctival scrapings and again cultured and passaged in eggs.

The major part of the expense of this research was met by the Ophthalmic Research Institute of Australia. The West Australian Public Health Department, and the Victorian Eye and Ear Hospital, Melbourne, also gave grants. It is a pleasure to acknowledge our very great indebtedness to our two volunteers, more especially to the enthusiastic and co-operative patient who received the virus inoculation, and, thereby, suffered no little pain and discomfort and very considerable restriction upon her normally active life. We should also like to thank Dr. Archie Anderson of Melbourne who was wholly responsible for obtaining the volunteers. Finally, we are much indebted to Miss J. Jenkyn who performed the complement-fixation tests and to Mr. T. Cottier, Clinical Photographer, Victorian Eye and Ear Hospital, for the illustrations.

\section{REFERENCES}

COllier, L. H., and SowA, J. (1958). Lancet, 1, 993.

Gilkes, M. J., Smith, C. H., and Sowa, J. (1958). Brit. J. Ophthal., 42, 473.

MANN, I. (1954a). "Ophthalmic Survey of the Kimberley Division of Western Australia". (West Australian Government Publication.) Government Printer, Perth. (1954b). "Ophthalmic Survey of the Eastern Goldfields Area of Western Australia". (Western Australian Government Publication.) Government Printer, Perth. (1956). "Ophthalmic Survey of the South-West Portion of Western Australia". (West Australian Government Publication.) Government Printer, Perth.

MEYER, K. F., and EDDIE, B. (1956). "Diagnostic Procedures for Virus and Rickettsial Diseases", 2nd ed., p. 409. American Public Health Association, New York City.

MORI, N. (1958). Amer. J. Ophthal., 45, 423.

PERRET, D., and MANN, I. (1960). Brit. J. Ophthal., 44, 503.

T'Ang, F.-F., Chang, H.-L., Huang, Y.-T., and WANG, K. D. (1957). Chin. med. J., 75, 429. 